University of Wollongong

Research Online

Faculty of Informatics - Papers (Archive)

Faculty of Engineering and Information

Sciences

2007

\title{
A Survey on Control Separation Techniques in Multi-Radio Multi-channel MAC Protocols
}

Jerry Chun-Ping Wang

University of Wollongong, jerryw@uow.edu.au

Mehran Abolhasan

University of Wollongong, mehran.abolhasan@uts.edu.au

Farzad Safaei

University of Wollongong, farzad@uow.edu.au

D. Franklin

University of Wollongong, daniel_franklin@uow.edu.au

Follow this and additional works at: https://ro.uow.edu.au/infopapers

Part of the Physical Sciences and Mathematics Commons

\section{Recommended Citation}

Wang, Jerry Chun-Ping; Abolhasan, Mehran; Safaei, Farzad; and Franklin, D.: A Survey on Control Separation Techniques in Multi-Radio Multi-channel MAC Protocols 2007.

https://ro.uow.edu.au/infopapers/644

Research Online is the open access institutional repository for the University of Wollongong. For further information contact the UOW Library: research-pubs@uow.edu.au 


\title{
A Survey on Control Separation Techniques in Multi-Radio Multi-channel MAC Protocols
}

\begin{abstract}
The rapid diminishing in the cost of commodity wireless hardware in recent years has prompted the use of multiple radios to improve the capacity of wireless networks. However, the research has shown that the improvement obtained from using multiple radios does not solely depend on the number of radios, but primarily on how these radios can be integrated in a constructive manner. A common way of integration multiple radios is to use a dedicated radio for control. To date, a number of multi-radio MAC protocol are employing a dedicated radio to control and coordinate the other radios, though the approaches are varied from one to another. In this paper, the control separation techniques in the multi-radio multi-channel MAC have been surveyed, and a classification of control separation techniques is provided. Moreover, this study points out the open research issues and intends to spark new interests and developments in this field.

\section{Disciplines}

Physical Sciences and Mathematics

\section{Publication Details}

This conference paper was originally published as Wang, JCP, Abolhasan, M, Safaei, F, Franklin, D, A Survey on Control Separation Techniques in Multi-Radio Multi-channel MAC Protocols, 7th International Symposium on Communications and Information Technologies ISCIT 2007, 17-19 Oct, 854-859.
\end{abstract}




\title{
A Survey on Control Separation Techniques in Multi-Radio Multi-channel MAC Protocols
}

\author{
J. C.-P. Wang, M. Abolhasan, F. Safaei, D. Franklin \\ Telecommunications and Information Technology Research Institute \\ University of Wollongong,Wollongong NSW 2522 Australia \\ Tel: +61-2-4221-4398, Fax: +61-2-4221-3277 \\ E-mail: $\{$ jcpw942,mehrana,farzad,danielf $\} @$ uow.edu.au
}

\begin{abstract}
The rapid diminishing in the cost of commodity wireless hardware in recent years has prompted the use of multiple radios to improve the capacity of wireless networks. However, the research has shown that the improvement obtained from using multiple radios does not solely depend on the number of radios, but primarily on how these radios can be integrated in a constructive manner. A common way of integration multiple radios is to use a dedicated radio for control. To date, a number of multi-radio MAC protocol are employing a dedicated radio to control and coordinate the other radios, though the approaches are varied from one to another. In this paper, the control separation techniques in the multi-radio multi-channel MAC have been surveyed, and a classification of control separation techniques is provided. Moreover, this study points out the open research issues and intends to spark new interests and developments in this field.
\end{abstract}

\section{INTRODUCTION}

The recent proliferation of wireless system has prompted the commoditization of RF transceivers whose prices have rapidly diminished in the past decade. As a result, it becomes feasible to employ multiple inexpensive wireless transceivers in a wireless device, and this can be traced to the development of multi-channel multi-radio ad hoc/mesh networks. For instance, Raniwala and Chieuh [1] proposed a multi-channel wireless mesh network architecture called Hyachinth which equips each mesh node with multiple 802.11 network interface cards.

However, the fact that each node equips with multiple transceivers imposes the trade-off between two conflicting objective: channel diversity and node connectivity. The use of multiple radios on one hand offers several non-overlapping channels for radios to operate on. This would improve the parallelism of network flows, thereby improving network capacity. On the other hand, since both sender and receiver must tune to the same channel in order to communicate, the network connectivity may be partitioned into several channel groups as each interface may switch to different channel. Thus, Ko et. al. [2] suggested one interface of each multi-radio node should be dedicated to a channel that is common to all nodes. This would ensure the connectivity between neighbors can be preserved.

Furthermore, according Design for Separation proposed Balh et. al. [3], it suggests that the control and data traffic should be assigned to different radios and operated in separate space and time. Interestingly, consider the nature of control and data flows in MAC protocols, the control message informs neighbors in vicinity (point-to-multipoints) about the subsequent point-to-point data transmission. Therefore, intuitively, the control separation is a common technique used in multiradio systems.

To date, there have been a number of MAC proposals featuring out-of-band control, though the approaches varied from one to another and there is no clear classification between these protocols. Hence, it is important to highlight the use of control separation technique and the improvement that it can achieve. In this paper, we surveyed existing multi-channel MAC featuring control separation and provide a classification of these multi-channel protocols based on their purpose of separation. We then identify the key advantages of each class. In addition, we pinpointed some deficiencies in these protocols which might spark new interests in the future multi-radio multi-channel MAC design.

The rest of this paper is organized as follows. Section 2 gives the description of the existing multi-channel MAC protocols. Section 3 summarizes the key features of these protocols and outlines the deficiencies of these MAC protocols. Finally, Section 4 concludes this paper.

\section{Description of Protocols}

There are many variations on multi-radio multi-channel MAC protocols that features control separation. In this section, we provide a protocol classification based on the functionalities of each protocol. We then describe the representative protocols of different classes.

\section{A. Principles of Operations}

The multi-radio multi-channel MAC protocols that use control separation technique to serve many different purposes: some exploit the multi-channel diversity provided by multiradios to enable simultaneous data transmission; some use separate channel to reserve future data usage while current data channel is occupied; some use separate signalling to address complete solution to hidden terminal problems. In this paper, we divide the multi-channel MAC protocol into four major classes. The basic packet operation of each class is shown in Figure 1.

In the subsequent sections, we describe the following control separation classes and the principle of their operations: 


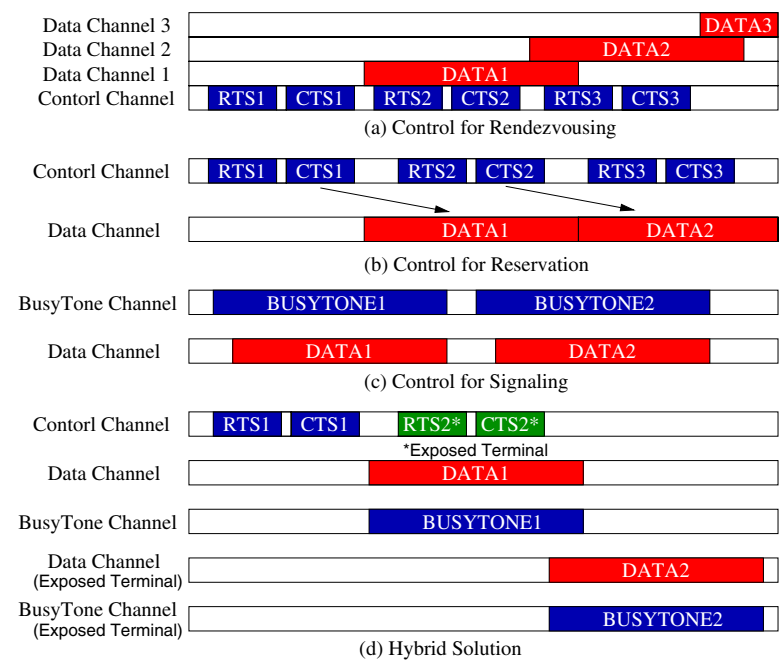

Fig. 1. Basic operation of different multi-channel MAC protocols

- Common Control Channel for Rendezvous: The control channel is used for arranging multiple simultaneous transmissions over multiple data channels.

- Common Control Channel for Reservation: The control channel is used for reservation and acquisition of future data channel access.

- Common Control Channel for Signalling: The control channel is used to emulate collision detection function as that in wired system.

- Hybrid Common Control Channel Techniques: A combination of aforementioned techniques or alternative approaches.

\section{B. Common Control Channel for Rendezvous}

Many multi-channel MAC protocols [4], [5], [6], [7], [8], [9] assign one dedicated control channel to enable the other interfaces to switch and transmit on pre-arranged channel from control channel. These protocols typically consist of a least two or more radio interfaces: one interface has to be tuned to a fixed common channel for control purposes, while the other interfaces can be dynamically switched between the remaining channels for data exchange.

Figure 1a illustrates the operation of rendezvous-based protocol. The dedicated control channel used in these protocols enables a node to arrange a rendezvousing data channel with its communicating counterpart. During every communication, the nodes must use control interface to exchange control messages in order to acquire the common data channel for exchanging data. The arrangement made by two communicating counterparts can also be heard by other nodes as the reference for the future data channel arrangement decision. Based on the information obtained from control interface, the data interface will be dynamically assigned as the common data channel and commence actual data transfer on the agreed channel.
Dynamic Channel Assignment (DCA) [4] is one of such rendezvousing method. In DCA protocol, each node manages two lists, namely: Channel Usage List (CUL) and Free Channel List (FCL) for the lists of busy and idle channels respectively. The main idea of DCA is that for every communication, a sender must initiate an RTS which piggybacks its CUL to the receiver. The receiver compares its FCL with the CUL from sender to select a data channel (if any) for the subsequent communication and reply a CTS with additional channel selection information. Upon receiving CTS from receiver, the sender will send a reservation (RES) packet to inform nearby nodes about reservation of the selected channel. Finally, both sender and receiver will switch their data interface to the selected channel, and commence data transmission.

In the subsequent work of DCA, Wu et. al. integrate the concept of power control into DCA protocol. This new protocol is known as Dynamic Channel Assignment with Power Control (DCA-PC) [5]. In DCA-PC, the protocol maintains an additional list called POWER which specifies the power level that should be used to transmit the data. The value of POWER can be dynamically adjusted as the node continuously monitoring the communication around itself on the control channel. Similar to DCA, DCA-PC goes through the same RTS/CTS/RES dialog to acquire a common data channel. When a data transmission is commenced on data interface, the sender will transmit the data with the power level that is specified on POWER list, thereby reducing energy consumption and signal interference.

Jain et. al. [6] identified the deficiency of DCA in which the data channel is randomly selected from a pool of free channels, and there is no clear indication on whether the best channel has been selected. Thus, they proposed a Receiver-Based Channel Selection (RBCS) scheme to support the functionality of DCA. Upon each successful reception of RTS along with a list of available channels, RBCS selects the best channel with least received power according to its own sensing if there is any. Once both sender and receiver have reached the consensus, data transmission will begin on agreed data channel.

Similarly, Hung et. al. also proposed Dynamic Private Channel (DPC) [7] that consists of one multicast control channel $(\mathrm{CCH})$ and multiple unicast data channels (DCHs). DPC is a connection-oriented protocol, if two nodes intend to communication, the sender will initiate the setup process by specifying a selected DCH in an RTS frame and sending to the receiver through $\mathrm{CCH}$. Once the RTS is received by receiver, the receiver will reply a Reply to RTS (RRTS) to accept the selected DCH or suggest alternative DCH. This process will continue until both sides reach an agreement on common DCH. Once channel negotiation comes to the end, the data exchange will commence with receiver sending out CTS to sender on selected DHC, and DATA-ACK exchange thereafter.

Liu et. al. [8] proposed an Adaptive Acquisition Collision Avoidance (AACA) protocol for ad hoc network. AACA resembles a typical rendezvousing scheme with one control channel and several data channels. Within AACA, there are 
three variations of AACA, they are - AACA with Single Designated Transmission Channel (AACA-SDT), AACA with Multiple Designated Transmission Channels (AACA-MDT) and AACA with Receiver Designated Transmission Channel (AACA-RDT) respectively, and these variations can be differentiated by the way of their data channel selection. In AACASDT, the sender makes the initiative on channel selection, and attaches the selected channel information to the RTS packet. The decision on whether the receiver agrees on the selected channel can be determined through reception of receiver's CTS packets. The AACA-MDT on the other hand is yet another version of DCA [4] - the list of free channels at the sender is piggybacked on an RTS packet, the receiver randomly selects a channel from the list, and encapsulates the channel information in CTS. If sender agrees with the channel selection, a sending acknowledgement (SACK) will be broadcast to inform nearby neighbors about the decision. Conversely, the channel selection decision in AACA-RTS is triggered by the receiver. The receiver selects a data channel from its viewpoint and relevant information is enclosed in a CTS packet. The channel agreement from sender is done through a sending acknowledgement (SACK).

Hsu et. al. explored the cognitive radio context and proposed a CSMA/CA based cognitive MAC protocol which is known as Statistical Channel Allocation MAC (SCA-MAC) [9]. Similar to other common channel rendezvousing schemes, SCA-MAC utilizes a common channel for initiating hand-shaking, while DATA/ACK messages are transmitted over data channel. The control channel SCA-MAC employs CSMA/CA scheme in which the node is permitted to transmit when the contention window expires and the channel remains idle. Thus, when the node is ready to transmit, the transmitting node sends a Control-channel-Request-To-Send (CRTS) message. Upon receiving CRTS, the receiving node exploits its own statistics of spectrum usage and the current status to allocate the channel for data transmission, and then replies with a Control-channelClear-To-Send (CCTS) message which contains the information of the best communication opportunity. Renegotiation is required if CRTS/CCTS can not be successfully exchanged or both sender and receiver do not have sufficient choice to make mutual agreement. Once CRTS/CCTS have been successfully exchanged, both sender and receiver will tune to agreed data channel, and then commence data transmission on data channel.

\section{Common Control Channel for Reservation}

A few proposals have considered the use of separate control channels for contention resolution and channel reservation to improve the transmission over data channel [10], [11], [12], [13], [14]. In contrast to rendezvous-based approach, reservation-based protocols do not exploit parallel transmission of data. Instead, it explores the enhancement over standard IEEE 802.11 DCF handshake with separate control channels. The protocols in this class typically feature only one data channel and one or more control channels. The control channels are used for exchanging control messages and facilitating data transmission over data channel. As a result control traffic can be stripped away from data traffic, thereby reducing control packet collision and its associate overhead. The protocols also enable control packets to reserve data channel in advance while data channel is occupied, this would avoid backoff overhead caused by contention resolution at the start of data transmission.

The operation of reservation based approach is shown in Figure $1 \mathrm{~b}$. The protocols in general follow standard RTS-CTSDATA-ACK exchange, though RTS-CTS and DATA-ACK are operated in different band and can be working in parallel. That is to say, while current data is transmitting on data channel, at the same time, the contention resolution of subsequent data transmission can be arranged. Thus, as soon as the current data transmission is completed, the next data transmission can commence immediately.

Yang et. al. [10] introduced a total pipelining strategy for IEEE 802.11 DCF using two channels called Dual Channel Pipelined Scheduling (DCPS). DCPS divides the channel into two sub-channels: a control channel and a data channel. The control channel is used for random backoff duration, collision detections, and RTS/CTS handshake; the data channel on the other hand is used for DATA/ACK exchange. The main feature of DCPS is that the overhead associated with contention resolution can be hidden (or partially hidden) by processing contention for next packet transmission in parallel with current packet transmission. However, the protocol has to ensure the RTS/CTS exchange for next data transmission must complete before current data transmission finishes, and subsequent RTS/CTS exchange can not begin until current data transmission terminates, otherwise, the efficiency of pipelining will be significantly reduced.

Similarly, Li et. al. [11] proposed a MAC protocol using a separating control channel (MAC-SCC), in which both control and data channels manage their own Network Allocation Vector (NAV) respectively. The protocol assumes that the bandwidth of data channel is much greater than control channel, and the data transmission follows standard IEEE 802.11 RTS-CTS-DATA-ACK four-way handshake. During the data transmission, all frames can be transmitted through data channel, while the control channel can only be used to transmit RTS or CTS frame. MAC-SCC primarily uses data channel for transmission whenever the channel is free. If data channel is busy, control channel can be use to schedule subsequent transmission on data channel. In other word, the contention of next data transmission can be resolved during the transmission of the current data frame. The exchange of RTS and CTS frame on the control channel can be used to extend NAV of the data channel on the nearby nodes. Thus, as soon the data channel is released from current transmission, the next data transmission can proceed immediately.

Zhang et. al. [12] indicated that the use of separate control channel for RTS/CTS will improve the performance of MAC protocol by keeping virtual carrier sensing while the node is in the transmitting state. This in effect would allow the nodes to be more aware of the channel state, thereby having more 
capability of initiating new transmission or responding to other nodes on the free data channel. Based on separating RTS/CTS control, Zhang et. al. proposed a new MAC protocol called Dual Control Channel based MAC (DCC-MAC) which further deploys a second control channel which is used exclusively for ACK transmission. Thus, two control channels are being used - one for RTS/CTS and the other for ACK respectively. The key advantage of using separate ACK reply contributes to the realization of unidirectional transmission on data channels. As a result, the frequent collision between data and ACK messages will be greatly reduced, and further improve the throughput.

Tantra et. al. [13] explored the use of a separate low-rate control channel to improve the performance of a high ratedata channel in an infrastructure-based wireless network, and proposed a new MAC layer scheduling scheme called Out-ofBand Signalling (OBS). Unlike other existing multi-channel protocols that divide total available bandwidth into two separate control and data sub-channel, OBS uses a separate unused channel operating at lower rate to schedule the actual data transmission on the higher rate channel. During each data transmission, a RTS frame will be transmitted to Access Point (AP) using DCF. Upon the reception of RTS frame at AP, the AP will then schedule the data transmission to the next PCF period for exchanging data and acknowledgement. This mechanism exploits the advantage of less overhead imposed by PCF, and resolves PCF synchronization by employing of outof-band DCF control. As a result, it improves the performance by mitigating costly idle periods and transmission collision on higher rate data channel.

Kyasanur et. al. have indicated that the use of OBS is restricted to infrastructure-based network, therefore proposing a novel Control Channel-based MAC protocol $\left(\mathrm{C}^{2} \mathrm{M}\right)$ [15] that features out of band control for multi-hop networks. $\mathrm{C}^{2} \mathrm{M}$ splits IEEE 802.11 DCF by operating the control portion of MAC protocol over a low-frequency, low-data rate, and long-range channel, while the data portion is operated over high-frequency, high data rate, and short-range channel. $\mathrm{C}^{2} \mathrm{M}$ highlights the use of small portion of spectrum at lower frequency to improve the performance of data transmission on the higher rate channel by allowing simultaneous channel contention resolution and data transmission. Further, it also exploits the advantage of longer transmission range at control channel in reducing the effect of hidden terminals on data channel.

\section{Common Control Channel for Signalling}

The third control separation approach - the common control channel for signalling is often referred as a tone-based approach. In this scheme, one or more separate narrow bandwidth radios are used to empower mobile hosts to send busy tone to emulate the collision detection function as that in wired network. The basic operation of tone-based approach is depicted in Figure 1c, instead of providing conventional RTS-CTS-DATA-ACK handshake, the busy tone serves two purposes one is collision avoidance and the other one is acknowledgement. The busy tone is a small pulse that occupies the control channel for duration of each data transmission. This, in effect, provides continuous physical carrier sensing to inform nearby nodes about the ongoing transmission on data channel. The nodes are not permitted to transmit any data if the busy tone is sensed. Upon the completion of data transmission, the busy tone will be terminated in order to inform the availability of data channel.

The use of busy tone to combat the hidden-terminal problems of CSMA has been appeared in a very early study of Busy Tone Multiple Access (BTMA) protocol proposed by Tobagi and Kleinrock [16]. BTMA is an infrastructure-based protocol which divides the channel into a message channel and the busy-tone channel. BTMA proposes that whenever a centralized base station senses a busy data channel, it transmits a busy tone over the busy tone channel to prevent nearby nodes from transmitting. Since, the base station is a centralized entity and is in line of sight of all terminals, each terminal can sense the busy-tone channel and determine the current state of data channel.

$\mathrm{Wu}$ and $\mathrm{Li}$ [17] proposed a receiver initiated busy-tone multiple access (RI-BTMA) protocol for packet-radio network. In this scheme, the sender transmits a preamble packet (eg. RTS) to the receiver prior to the data transmission. When the receiver successfully obtains the preamble and the data channel is available. The receiver will initiate a busy tone in a separate channel to inform nearby node that they should backoff. So the correct source can always be notified that it can proceed with transmission of data packet.

In the subsequent work of tone-based approach, Haas and Deng [18] designed a Dual Busy Tone Multiple Access (DBTMA) for ad hoc network which uses RTS requests and two out-of-band busy tones. This scheme features two busy tone signals, namely "Transmit-Busy-Tone" $\left(B T_{t}\right)$ and "Receive-Busy-Tone" $\left(B T_{r}\right) . B T_{t}$ prevents the exposed node becoming new receiver, so RTS can be promptly delivered to all nearby nodes. On the other hand, $B T_{r}$ is used to prevent the hidden terminals from becoming new transmitter, and avoid collision between control and data packets. In addition, the $B T_{r}$ can only be initiated upon successful reception of RTS packet, thus, $B T_{r}$ can replace CTS and serve as an acknowledgement to the RTS packets.

\section{E. Hybrid Common Control Channel Techniques}

From the previous discussion, it is clear that different classes of protocols offer unique advantages in one way or another. Thus, there are many protocols that combine these features and form a new hybrid protocol. For instance, Power Aware Multi-Access protocol with Signalling (PAMAS) [19] utilizes a separate control channel for exchanging RTS/CTS. The length of the upcoming transmission is attached in both RTS and CTS, and the neighboring nodes that overhear RTS or CTS messages can therefore go into sleep mode for the duration of the transmission, thus enabling power saving capabilities. Further, an extra busy tone signal is used to ensure the hidden terminal problem will not occur. 
Zhai et. al. proposed a new dual-channel MAC protocol (DUCHA) [20] for multi-hop mobile ad hoc network. This protocol utilizes dual-channel for control and data packets, where RTS and CTS are transmitted over control channel. The operation of DUCHA is shown in Figure 1d, from the figure, it has shown that the used of separate control channel allows RTS/CTS to be exchanged at exposed node when the other exposed terminal is transmitting data. As a result, the exposed terminal problems could be resolved, and spatial reuse could be maximized. In addition, DUCHA exploits the use of out-ofband receiver based busy tone to address the hidden terminal problem.

Ma et. al. proposed a Cognitive Radio (CR) based Dynamic Open Spectrum Sharing (DOSS) MAC [21], which consists of three operational channels: a busy tone channel, a control channel, and a data channel. DOSS enables CR devices to identify and negotiate potential data band through the common control channel, and then send data through the continuous fraction of the data band in which both sender and receiver agree. Moreover, the hidden and exposed terminal problem can be alleviated by raising the busy-tone signal in the busy-tone channel.

Comparably, Yang et. al. [22] design a "Double Sense Multiple Access" (DSMA) scheme based on DBTMA and separate RTS/CTS control. DSMA maintains two out-of-band busy tone, one control channel for RTS/CTS, and one for data transmission. Yang et. al. have identified the problems that the busy tones can not specify the source of busy tone; the collision may occur as multiple senders have sensed the busy-tone and transmitting data at same time. Thus, DSMA employs an additional double sense mechanism which senses the receive-busy-tone prior and after the completion of RTS in order to ensure the authenticity of the busy tone. The node must ensure the receive-busy-tone only appears in second sense, otherwise, the receive-busy-tone could be reserved for some other nodes. In this way, no packet collision in data channel can be guaranteed.

\section{Summary \& DiscusSiON}

The summary of these multi-channel protocols is depicted in Table I. By observing the characteristics of rendezvousbased protocols, it can be seen that rendezvous-based protocols exploit the multi-channel diversity and allow parallel transmission of data. In addition, this approach also solves the exposed terminal problem as the exposed node can use separate channel for data transmission. However, most protocols under these schemes do not consider the channel switching overhead which may incur when interface switches from one channel to another and the network may experience large switching overhead if frequent channel switching occurs.

In contrast, the reservation based scheme employs only one data channel and it uses one or more separate control channels to enable advanced contention resolution on the data channel in a way that the control and data are operating in a pipeline. That is, the next data transmission will be scheduled on the control channel while current data is transmitting over data channel. Since the next data transmission is pre-arranged, there will be no contention backoff after each data transmission. The next data transmission will be commenced as soon as current transmission completes. As a result, it takes the advantage of seamless data transmission over data channel and achieves high data channel utilization. However, the major drawback in this scheme though is that the exposed terminal problem still remains unsolved as only one data channel used at a time and the multi-channel diversity provided by multiple radios will not exploited.

The tone-based scheme identifies that the conventional RTS/CTS handshake does not solve hidden terminal problem completely as RTS may collide with other RTS. Thus, providing a busy tone would allow nearby nodes to be constantly aware of channel condition. However, since the busy-tone is a pulse over time domain, and it does not carry any information, this provides insufficient information for the sender on whether is delivered properly.

The hybrid approach combines the advantage of aforementioned schemes to overcome the problems that may occur in any unicast transmission. This type of approach in general relies on exchanging control information over one or more control channels, and it is essential to keep all control channels synchronized before commencing data transmission; failures in any one of the control channels would result in longer control overhead or packets drops.

One of the common drawbacks of the control separation technique is the control channel saturation. Consider that all data transmission require to be initiated through the exchange of control messages on the control channel, it is inevitable that the control channel could become a potential bottleneck for the data transmission if the bandwidth of control channel is small. Once the control channel becomes saturated, the control message will not be successfully delivered. Besides, the condition of control channel does not necessary reflects on the data channel as two radios my hold different physical characteristic. Consequently, the other interfaces may not be fully utilized. One possible solution should be involved with more careful use of control channel such as "data train" proposed by Kyasanur et. al [15].

\section{CONCLUSION}

The use of a common channel for exchanging control message is a common implementation approach in multiradio systems. This study highlights the control separation technique and provides a broad overview of the multi-radio multi-channel MAC protocol employing such technique. We have presented a classification on these multi-radio multichannel MAC protocols and discussed the salient features of the representative protocols in each class. The separate control channel in general can coordinate the transmission over other channels. It also enables the additional pipelined controls over other channel to improve data transmission. However, as it has been pointed out in this paper that control separation technique may lead to control channel saturation and cause bottleneck. 
TABLE I

SuMmary OF CONTROL SEPARATION TECHNiQUES

\begin{tabular}{|c|c|c|c|c|c|c|c|c|c|}
\hline Approach & Class & \# of channels & Sol. to HT & Sol. to ET & Adv. Res. & Parallel Trans. & Pow. Con. & Broadcast & $\mathrm{Ad} \mathrm{Hoc}$ \\
\hline$\overline{\mathrm{DCA}}[4]$ & Rendezvous & $\geq 2$ & Yes $^{\mathrm{a}}$ & Yes & No & Yes & No & No & Yes \\
\hline DCA-PC[5] & Rendezvous & $\geq 2$ & Yes $^{\mathrm{a}}$ & Yes & No & Yes & Yes & No & Yes \\
\hline RCBS[6] & Rendezvous & $\geq 2$ & Yes $^{\mathrm{a}}$ & Yes & No & Yes & No & No & Yes \\
\hline DPC[7] & Rendezvous & $\geq 2$ & Yes $^{\mathrm{a}}$ & Yes & No & Yes & No & No & Yes \\
\hline $\mathrm{AACA}[8]$ & Rendezvous & $\geq 2$ & Yes $^{\mathrm{a}}$ & Yes & No & Yes & No & No & Yes \\
\hline SCA-MAC[9] & Rendezvous & $\geq 2$ & Yes $^{\mathrm{a}}$ & Yes & No & Yes & No & No & Yes \\
\hline DCPS[10] & Reservation & 2 & Yes $^{\mathrm{a}}$ & No & Yes & No & No & No & Yes \\
\hline MAC-SCC[11] & Reservation & 2 & Yes $^{\mathrm{a}}$ & No & Yes & No & No & No & Yes \\
\hline DCC-MAC[12] & Reservation & 3 & Yes $^{\mathrm{a}}$ & No & Yes & No & No & No & Yes \\
\hline $\mathrm{C}^{2} \mathrm{M}[15]$ & Reservation & 2 & $\mathrm{Yes}^{\mathrm{a}, \mathrm{b}}$ & No & Yes & No & No & No & Yes \\
\hline OBS[13] & Reservation & 2 & Yes & Yes & Yes & No & No & No & No \\
\hline ВТМА[16] & Signaling & 2 & Yes & No & No & No & No & Yes & No \\
\hline RI-BTMA[17] & Signaling & 2 & Yes & No & No & No & No & Yes & No \\
\hline DBTMA[18] & Signaling & 3 & Yes & No & No & No & No & Yes & Yes \\
\hline PAMAS[19] & Hybrid & 3 & Yes & No & No & No & Yes & No & Yes \\
\hline DUCHA[20] & Hybrid & 3 & Yes & Yes & No & No & No & No & Yes \\
\hline DOSS [21] & Hybrid & 3 & Yes & Yes & No & Yes & No & No & Yes \\
\hline DSMA[22] & Hybrid & 4 & Yes $^{\mathrm{a}}$ & No & No & No & No & No & Yes \\
\hline
\end{tabular}

${ }^{a}$ The collisions of RTSs still occur under these schemes.

${ }^{\mathrm{b}} \mathrm{C}^{2} \mathrm{M}$ exploits the longer transmission range of out-of-band radio, and is able to extend the range of RTS/CTS effect.

Therefore, more research is still needed in order to build a more robust and efficient multi-radio system.

\section{REFERENCES}

[1] A. Raniwala and T. cker Chiueh, "Architecture and algorithms for an ieee 802.11-based multi-channel wireless mesh network," in Proceedings of IEEE INFOCOM 2005., vol. 3, March 2005, pp. 2223-2234.

[2] B.-J. Ko, V. Misra, J. Padhye, and D. Rubenstein, "Distributed channel assignment in multi-radio 802.11 mesh networks," in Proceedings of IEEE Wireless Communication and Network Conference (WCNC 2007), 2007.

[3] P. Bahl, A. Adya, J. Padhye, and A. Walman, "Reconsidering wireless systems with multiple radios," SIGCOMM Computer Communication Review, vol. 34, no. 5, pp. 39-46, October 2004.

[4] S.-L. Wu, C.-Y. Lin, Y.-C. Tseng, and J.-L. Sheu, "A new multichannel mac protocol with on-demand channel assignment for multi-hop mobile ad hoc networks," in Proceedings of International Symposium on Parallel Architectures, Algorithms and Networks (I-SPAN), December 2000, pp. $232-237$.

[5] S.-L. Wu, Y.-C. Tseng, C.-Y. Lin, and J.-L. Sheu, "A multi-channel mac protocol with power control for multi-hop mobile ad-hoc networks," The Computer Journal, vol. 45, no. 1, pp. 101-110, 2002.

[6] N. Jain, S. R. Das, and A. Nasipuri, "A multichannel csma mac protocol with receiver-based channel selection for multihop wireless networks," in Proceedings of the 9th International Conference on Computer Communications and Networks (IC3N), Scottsdale, AZ, USA, October 2001, pp. 432-439.

[7] W.-C. Hung, K. Law, and A. Leon-Garcia, "A dynamic multi-channel mac for ad hoc lan," in 21st Biennial Symposium on Communications, Kingston, Canada, June 2002, pp. 31-35.

[8] K. Liu, T. Wong, J. Li, L. Bu, and J. Han, "A reservation-based multiple access protocol with collision avoidance for wireless multihop ad hoc networks," in Proceedings of IEEE International Conference on Communications, 2003. (ICC '03), vol. 2, May 2003, pp. 1119-1123.

[9] A. C.-C. Hsu, D. S. L. Wei, and C.-C. J. Kuo, "A cognitive mac protocol using statistical channel allocation for wireless ad-hoc networks," in Proceedings of IEEE Wireless Communication \& Networking Conference (WCNC), Hong Kong, March 2007.

[10] X. Yang, N. H. Vaidya, and P. Ravichandran, "Split-channel pipelined packet scheduling for wireless networks," IEEE Transactions on Wireless Communications, vol. 5, no. 3, pp. 240-257, March 2006.

[11] Y. Li, H. W. s, N.-F. Tzeng, D. Perkin, and M. Bayoumi, "MACSCC: a medium access control protocol with separate control channel for reconfigurable multi-hop wireless networks," IEEE Transactions on Wireless Communications, vol. 5, no. 7, pp. 1805-1817, July 2006.
[12] J. Zhang, Y. Wang, and J. Wang, "DCC-MAC: a new mac protocol for ad-hoc networks based on dual control channel," in Proceedings of 14th IEEE Proceedings on Personal, Indoor and Mobile Radio Communications (PIMRC), vol. 2, September 2003, pp. 1341- 1345.

[13] J. W. Tantra, C. H. Foh, and B. S. Lee, "An efficient scheduling scheme for high speed ieee 802.11 wlans," in Proceedings of IEEE 58th Vehicular Technology Conference, (VTC 2003-Fall), vol. 4, October 2003, pp. 2589-2593.

[14] P. Kyasanur and N. H. Vaidya, "Routing and interface assignment in multi-channel multi-interface wireless network," in Proceedings of IEEE Wireless Communications and Networking Conference (WCNC), vol. 4, March 2005, pp. $2051-2056$.

[15] P. Kyasanur, J. Padhye, and P. Bahl, "On the efficacy of separating control and data into different frequency bands," in Proceedings of 2 nd International Conference on Broadband Networks, vol. 1, October 2005, pp. 602-611.

[16] F. A. Tobagi and L. Kleinrock, "Packet switching in radio channels: Part ii - the hidden terminal problem in carrier sense multiple-access and the busy-tone solution," IEEE Transaction on Communications, vol. COM-23, no. 12, pp. 1417-1433, December 1975.

[17] C. Wu and V. Li, "Receiver-initiated busy-tone multiple access in packet radio networks," SIGCOMM Comput. Commun. Rev., vol. 17, no. 5, pp. 336-342, 1988

[18] Z. J. Haas and J. Deng, "Dual busy tone multiple access (DBTMA)-a multiple access control scheme for ad hoc networks," IEEE Transactions on Communications, vol. 50, no. 6, pp. 975 - 985, June 2002.

[19] S. Singh and C. S. Raghavendra, "Pamas - power aware multi-access protocol with signalling for ad hoc networks," SIGCOMM Comput. Commun. Rev., vol. 28, no. 3, pp. 5-26, 1998.

[20] H. Zhai, J. Wang, and Y. Fang, "Ducha: A new dual-channel mac protocol for multihop ad hoc networks," IEEE Transactions on Wireless Communications, vol. 5, no. 11, pp. 3224 -3233, November 2006.

[21] L. Ma, X. Han, and C.-C. Shen, "Dynamic open spectrum sharing mac protocol for wireless ad hoc networks," in Proceedings of First IEEE International Symposium on New Frontiers in Dynamic Spectrum Access Networks (DySPAN), November 2005, pp. 203-213.

[22] Y. Yang, F. Huang, X. Gu, M. Guizani, and H.-H. Chen, "Double sense multiple access for wireless ad hoc networks," in QShine '06: Proceedings of the 3rd international conference on Quality of service in heterogeneous wired/wireless networks. New York, NY, USA: ACM Press, 2006, p. 9. 